\title{
A Rare Late Term Complication of Vascular Surgery: True Com- mon Femoral Artery Aneurysm
}

\author{
Ali Buturak ${ }^{1 *}$, Ulaankhuu Batgerel ${ }^{2}$ and Duhan Fatih Bayrak ${ }^{3}$ \\ ${ }^{1}$ Department of Cardiology, Florence Nightingale Group Kadıkoy and Atasehir Hospitals, Istanbul, Turkey \\ ${ }^{2}$ Department of Cardiology, Acibadem Kadıkoy Hospital, Istanbul, Turkey \\ ${ }^{3}$ Department of Cardiovascular Surgery, Acibadem University, Istanbul, Turkey
}

*Corresponding author: Ali Buturak, Department of Cardiology, Florence Nightingale Group Kadikoy and Atasehir Hospitals, Istanbul, Turkey, Tel:+905052702856, Fax:+902164969668

\begin{abstract}
Femoral artery aneurysm is a rarely seen peripheral aneurysm which may lead to thrombosis, embolism and fatal rupture in untreated cases. Although femoral artery aneurysm is usually caused by atherosclerosis or chronic inflammation, aneurysm formation in sites of anastomosis, grafts or endarterectomy territory may occur at late term. A 57-yearsold man presenting with stable angina pectoris was admitted for coronary angiography with documented ischemia in exercise stress test. The patient had a history of left femoral thromboendarterectomy and venous patch plasty five years ago. Physical examination revealed a pulsatile mass in the left groin. Coronary angiography revealed a distal circumflex artery lesion and follow up with optimal medical treatment was planned for coronary artery disease. Afterwards, a lower extremity arteriography was performed which indicated a true aneurysm of the left common femoral artery (CFA). The aneurysm was at the site of previous endarterectomy and patch plasty pointing out an iatrogenic etiology. A multidetector computed tomography (MDCT) angiography was performed to rule out any other arterial aneurysms and confirm the relation of the aneurysm and deep femoral artery bifurcation. Treatment with surgical repair was planned since the aneurysm was covering the bifurcation and had iatrogenic etiology.
\end{abstract}

\section{Keywords}

Femoral, Aneurysm, Complication

\section{Learning Objective}

To diagnose and manage femoral artery aneurysms which are rarely seen peripheral aneurysms.

\section{Introduction}

Femoral artery aneurysms are uncommon entities which may lead to complications such as embolism, thrombosis, rupture and result in increased mortality and morbidity $[1,2]$. Although atherosclerosis is the most common cause of femoral artery aneurysms, previous vascular reconstructive surgery such as endarterectomy and/or patch plasty may lead to aneurysm progression in femoral artery territory [3,4].

We report a left common femoral artery true aneurysm involving the deep femoral artery bifurcation in a patient with a history of left femoral thromboendarterectomy and venous patch plasty five years ago.

\section{Case Report}

A 57-years-old man with stable angina pectoris was admitted to coronary angiography since exercise stress test revealed ischemic electrocardiographic findings. Type 2 diabetes mellitus and hypertension existed in addition to active smoking. He had a history of left femoral artery thromboendarterectomy plus patch plasty for intermittent claudication five years ago. A pulsatile mass was palpated in the left groin in our current physical examination, for this reason, arterial duplex ultrasound was performed and a left common femoral artery (CFA) aneurysm (1.7 cm in width) was detected. Then a right transradial coronary angiography was performed which indicated a $75 \%$ stenosis of distal left circumflex artery. In the same procedure, a lower extremity arteriography

Citation: Buturak A, Batgerel U, Bayrak DF (2018) A Rare Late Term Complication of Vascular Surgery: True Common Femoral Artery Aneurysm. Int J Clin Cardiol 5:121. doi.org/10.23937/23782951/1410121

Accepted: September 22, 2018; Published: September 24, 2018

Copyright: (C) 2018 Buturak A, et al. This is an open-access article distributed under the terms of the Creative Commons Attribution License, which permits unrestricted use, distribution, and reproduction in any medium, provided the original author and source are credited. 
was performed which revealed a type 2 left CFA aneurysm involving the deep femoral artery (DFA) ostium (Figure 1). The CFA aneurysm was at the site of previous endarterectomy and patch plasty pointing out an iatrogenic etiology. A multidetector computed tomography (MSCT) angiography was performed to scan other arterial aneurysms and confirm the relation of the aneurysm and DFA bifurcation. MSCT angiography views

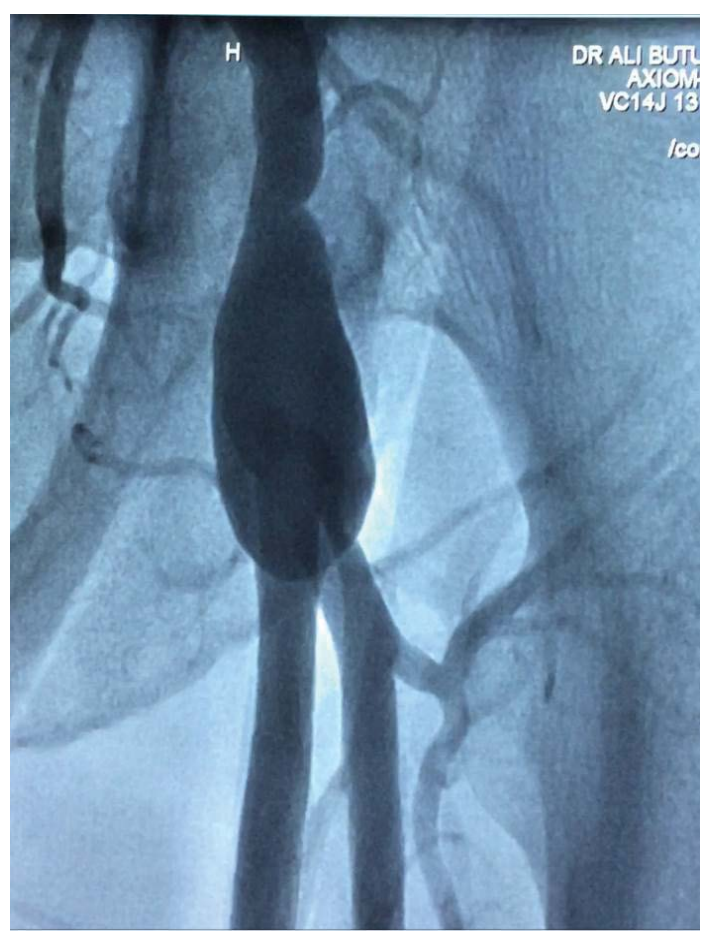

Figure 1: Transradial aortography indicating a left common femoral artery aneurysm involving superficial and deep femoral artery bifurcation. revealed that CFA aneurysm $(1.7 \mathrm{~cm}$ in width and $3.0 \mathrm{~cm}$ in length) was covering the ostium of DFA and superficial femoral artery (Figure 2a and Figure 2b). There were no other aneurysms in MSCT angiography views. After consulting the patient with the cardiovascular surgeons, duplex ultrasound follow-up was planned and CX lesion was treated with direct stenting via transradial access. Dual anti-platelet therapy with aspirin and clopidogrel, atorvastatin $40 \mathrm{mg}$, ramipril $2.5 \mathrm{mg}$ and metoprolol 50 mg once daily were given to the patient. Duplex ultrasound examination was planned for 6 months later.

\section{Discussion}

Femoral artery true aneurysms are the second most common peripheral arterial aneurysms following the popliteal artery aneurysms. Even though these aneurysms are uncommon, they have the potential of thrombosis, embolization or rupture especially in the cases of progressive aneurysmal dilatation $[1,2]$. The accurate incidence of the disease is unknown since the published articles regarding the incidence of true femoral artery aneurysms are case reports [5]. The common femoral artery is the most affected site even though isolated superficial and deep femoral arteries are rarely involved [2]. True femoral artery aneurysms are frequently bilateral and associated with other aneurysms in abdominal aorta or popliteal arteries [3]. CFA aneurysms are classified as type 1 and type 2 according to the relation of the aneurysm and CFA bifurcation [6]. Type 2 CFA aneurysm involves deep femoral artery ostium and femoral artery bifurcation as it is seen in the present case which complicates therapy options.
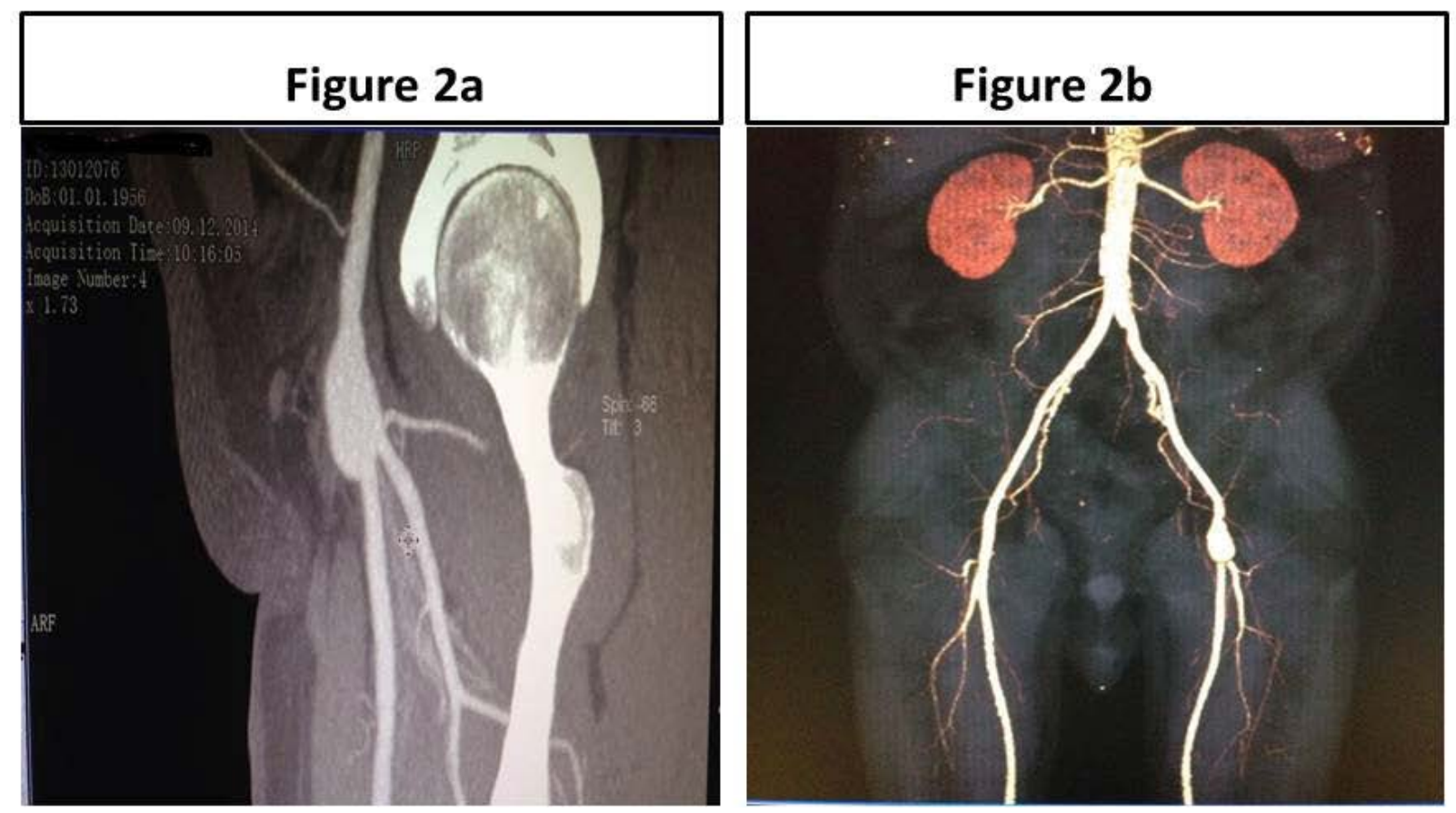

Figure 2: a) Sagittal view CT angiographic image demonstrates left common femoral artery aneurysm $1.7 \mathrm{~cm}$ in width involving deep femoral artery ostium and bifurcation; b) Frontal VRT image shows distal abdominal aorta, bilateral iliac, common femoral, deep femoral and superficial femoral arteries. Left common femoral artery aneurysm is seen and there is no other aneurysm formation in aorta. 
CFA aneurysms are usually asymptomatic in approximately $50 \%$ of the patients. A pulsatile mass in the affected groin may be the sole finding in asymptomatic cases. Acute limb ischemia caused by acute thrombosis is reported in $15 \%$ of the cases whereas distal embolization occurs in $0-26 \%$ of the patients with a wide range of incidence. Rupture is a fatal complication of CFA aneurysms however it is rarely seen with an incidence varying between $10-14 \%$ [3]. Groin or anterior thigh pain and paresis in related muscle groups may manifest due to compression of adjacent nervous structures. The common femoral vein may also be affected which would cause lower extremity edema and venous stasis in extremely large aneurysms [7].

Atherosclerosis is the leading etiology in majority of the cases with predisposing risk factors such as smoking, male gender and advanced age $[1,2,7]$. Chronic mycosis and syphilis is may also involve the arterial wall and give rise to aneurysmal dilatation in the artery. Likewise, connective tissue disorders such as Behçet's disease and systemic lupus erythematosus may induce chronic arteritis which afterwards progresses to aneurysmal disease [8]. Femoral artery true aneurysms are also associated with iatrogenic etiology following vascular surgical procedures. Suture rupture, detachment of an anastomosed graft, inappropriate sized graft implantation, perioperative infections, hematoma and perioperative graft damage may lead to iatrogenic true aneurysm formation. Venous patch plasty and thromboendarterectomy may result in aneurysmal dilatation of the femoral artery as we observed in our patient five years later following the surgery [4]. Although CFA aneurysms with iatrogenic etiology usually appear about five years after the surgery, they may be seen at any time on long term follow up [4]. Symptomatic CFA true aneurysms and asymptomatic CFA aneurysms with a width greater than $2.5 \mathrm{~cm}$ should be treated with surgical reconstruction [9]. Asymptomatic smaller aneurysms with an iatrogen- ic etiology should be followed up by duplex ultrasound and/or MSCT angiography closely.

In conclusion, patients with a history of vascular reconstructive surgery including femoral thromboendarterectomy and patch plasty should be screened for true aneurysm formation at late term. Although these iatrogenic aneurysms are rarely seen, limb threatening complications such as acute thrombosis and embolization may occur if they are left untreated or not followed up.

\section{Conflict of Interest}

None.

\section{References}

1. Kolde E, Rocha MF, Franco FC, Ornato SJTA (1998) Ruptura de aneurisma de arteria femoral. Cir Vasc Angiol 14: 40-42.

2. Harbuzariu C, Duncan AA, Bower TC, Kalra M, Gloviczki P (2008) Profunda femoris artery aneurysm: Association with aneurysmal disease and limb ischemia. J Vasc Surg 47: 31-34.

3. Levi N, Schroeder TV (1997) Arteriosclerotic femoral artery aneurysms: A short review. J Cardiovasc Surg 38: 335-338.

4. Gaylis H, Dewar G (1990) Anastomotic aneurysms: Facts and fancy. Surg Annu 22: 317-341.

5. Mitchell ME, Carpenter JP (2001) Popliteal artery aneurysm. Current therapy in vascular surgery. $\left(4^{\text {th }}\right.$ edn $)$, Mossby, Missouri, USA, 341-345.

6. Cutler BS, Darling RC (1973) Surgical management of arteriosclerotic femoral aneurysms. Surgery 74: 764-773.

7. Arroyo-Bielsa A, Rodríguez-Montalbán Al, Sáinz-González F (1995) Aneurismas ateroescleróticos de la arteria femoral común. Angiología 5: 251-256.

8. Koç Y, Güllü I, Akpek G, Akpolat T, Kansu E, et al. (1992) Vascular involvement in Behçet"s disease. J Rheumatol 19: 402-410.

9. Rigdon EE, Monajjen N (1992) Aneurysms of the superficial femoral artery: A report of two cases and review of the literature. J Vasc Surg 16: 790-793. 